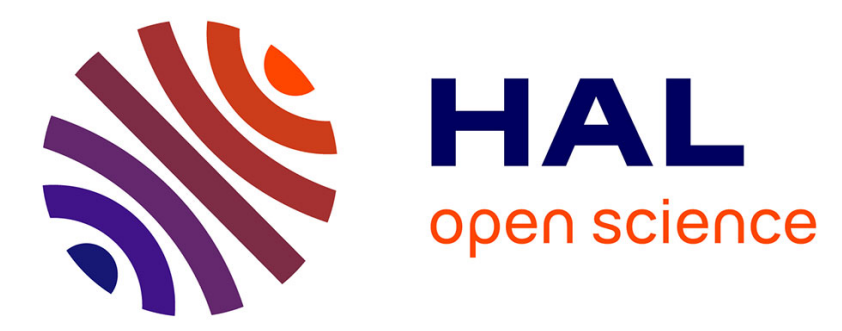

\title{
Three-dimensional modeling of earthquake generated acoustic waves in the ocean in simplified configurations
}

Jean Lecoulant, Claude Guennou, Laurent Guillon, Jean-Yves Royer

\section{To cite this version:}

Jean Lecoulant, Claude Guennou, Laurent Guillon, Jean-Yves Royer. Three-dimensional modeling of earthquake generated acoustic waves in the ocean in simplified configurations. Journal of the Acoustical Society of America, 2019, 146, pp.2113 - 2123. 10.1121/1.5126009 . hal-03319876

\section{HAL Id: hal-03319876 \\ https://hal.univ-brest.fr/hal-03319876}

Submitted on 13 Aug 2021

HAL is a multi-disciplinary open access archive for the deposit and dissemination of scientific research documents, whether they are published or not. The documents may come from teaching and research institutions in France or abroad, or from public or private research centers.
L'archive ouverte pluridisciplinaire HAL, est destinée au dépôt et à la diffusion de documents scientifiques de niveau recherche, publiés ou non, émanant des établissements d'enseignement et de recherche français ou étrangers, des laboratoires publics ou privés. 


\title{
Three-dimensional modeling of earthquake generated acoustic waves in the ocean in simplified configurations
}

\author{
Jean Lecoulant, ${ }^{1, a)}$ Claude Guennou, ${ }^{1}$ Laurent Guillon, ${ }^{2}$ and Jean-Yves Royer ${ }^{1}$ \\ ${ }^{1}$ Laboratoire Géosciences Océan, University of Brest and CNRS, Brest, 29200, France \\ ${ }^{2}$ Institut de Recherche de l'Ecole Navale, École Navale, Brest, 29200, France
}

(Received 8 November 2018; revised 19 July 2019; accepted 19 July 2019; published online 30 September 2019)

\begin{abstract}
The low-frequency $(4-40 \mathrm{~Hz})$ acoustic waves generated by undersea earthquakes are of great importance to monitor the low-level seismic activity associated with seafloor spreading ridges. To better understand the near-source interaction of seismic waves with the seafloor and the resulting generation of low-frequency acoustic waves, the wave propagation in a solid medium (the Earth's crust) and in the overlaying fluid medium (the ocean) were jointly simulated using a threedimensional (3D) spectral finite-element code (SPECFEM3D). Due to numerical limitations of 3D simulations, the focus was on simple model configurations with a $1 \mathrm{~Hz}$ source located below a Gaussian seamount or ridge. The simulated acoustic waves $(0-2.5 \mathrm{~Hz})$ propagate as Rayleigh modes and are affected by modal dispersion; their horizontal speed increases away from the source and reaches the sound speed about $140 \mathrm{~km}$ away. The amplitude of the generated acoustic waves is affected by the shape of the seafloor topography above the seismic source, as well as their travel times to hydrophones. Consequently, localization of the acoustic sources by trilateration from arrival times may be biased by 3D-effects, and thus the seismic/acoustic conversion zone may not match the epicenter. (C) 2019 Acoustical Society of America. https://doi.org/10.1121/1.5126009
\end{abstract}

[MP]

Pages: $2110-2120$

\section{INTRODUCTION}

The underwater seismic activity occurring at seafloor spreading ridges generates a large amount of low-frequency acoustic waves $(4-40 \mathrm{~Hz})$. In this paper, we model the generation and propagation of acoustic waves using the threedimensional (3D) numerical code SPECFEM3D. Since space and time resolution in 3D-modeling are rapidly limited by numerical resources, we used simplified model configurations and simulated very low-frequency acoustic waves $(0-2.5 \mathrm{~Hz})$. Despite these limitations, the generated acoustic waves resemble actual higher-frequency acoustic waves generated by submarine earthquakes.

Seismic waves generated by earthquakes propagate in the Earth's crust until they reach the sea-bottom. Depending on the local topography, they may convert to acoustic waves that propagate into the water column (Okal, 2008). These waves are known as $T$ waves (tertiary waves), because they may convert back, onshore, into seismic waves and arrive at seismographs after the $P$ (primary) and $S$ (secondary) waves. Seismic data acquired on-land generally provide no or little information on the low-level seismic activity at mid-ocean ridges (e.g., Bohnenstiehl et al., 2002) due to the rapid attenuation of seismic waves in the Earth's crust. This difficulty can be overcome by recording $T$ waves in the ocean with hydrophones, which takes advantage of the excellent acoustic properties of the ocean, since $T$ waves can propagate over very long-distances (beyond $1000 \mathrm{~km}$ ) with little attenuation.

For this reason, $T$ waves can improve our understanding of seafloor spreading ridges (e.g., Fox et al., 2001). For example,

${ }^{\text {a)} E l e c t r o n i c ~ m a i l: ~ j e a n . l e c o u l a n t @ u n i v-b r e s t . f r ~}$ the source of $T$ waves can be located by trilateration from their arrival times to an array of hydrophones. The assumption is that, for shallow earthquakes, the seismic/acoustic conversion area corresponds to the epicenter. Acoustic source-levels, inferred from $T$ wave amplitudes at the receivers, provide additional information allowing to compare events or infer sourcelevel/magnitude relationships. However, the hilly topography of spreading ridges may have 3D-effects on the generation of $T$ waves that may lead to a seismic/acoustic conversion area that may not correspond to the epicenter. In the same way, the received levels of a single event, after correction for propagation losses, generally differ among hydrophones. This may be due to the radiation pattern (i.e., focal mechanism) of the seismic source, but also to local 3D-effects of the seafloor topography.

To investigate these questions, we use a 3D spectral finite-element code (SPECFEM3D; Tromp et al., 2008) to simulate the propagation of a seismo-acoustic wave in a solid medium (the Earth's crust) and a fluid medium (the ocean), both modeled using an unstructured hexahedral mesh. A spectral element method presents many advantages for modeling the propagation of acoustic waves. Such a method lends itself to the study of seismic-acoustic conversion because it can finely model an interface with an arbitrary geometry. In contrast with commonly used acoustic codes, SPECFEM3D is not limited to a point source in the water column but can handle the whole surface exposed to seismic waves behaving like a surface acoustic source. Unlike ray-code modeling, limited to high-frequency acoustic waves, or modal-code modeling, limited to lowfrequency acoustic waves, the spectral element method is not limited to a single frequency-domain, and can thus 
handle very low-frequency seismic and acoustic waves without any a priori hypothesis on the type of propagation. Moreover, a time signal can be extracted at any point in the mesh, allowing to simulate "hydrophones" anywhere in the mesh.

This paper shows that, in simplified configurations reproducing an idealized ridge or an isolated seamount, SPECFEM3D is able to simulate the generation and propagation of very low-frequency acoustic waves. These simplified configurations highlight 3D-effects influencing the generation of acoustic waves and their consequences on the wave amplitudes and on the localization of an epicenter by trilateration. Section II presents the SPECFEM3D code used for modeling acoustic waves and the schematic configurations set. Section III presents the acoustic waves observed with such configurations, how they are generated, and how they propagate. Section IV presents the 3D-effects affecting the localization of the epicenter by using the travel times of acoustic waves.

\section{NUMERICAL MODELING}

\section{A. SPECFEM3D}

SPECFEM3D modeling is based on several simplifying assumptions that make it possible to solve the propagation equations in the crust and in the ocean. The Earth's crust is considered as a lossless linear elastic solid where the code solves the momentum conservation (Peter et al., 2011)

$$
\rho_{s} \partial_{t}^{2} \vec{u}^{s}=\vec{\nabla} \cdot \overline{\bar{\sigma}}+\vec{f},
$$

where $\rho_{s}$ is the density of the solid medium, $\vec{u}^{s}$ is the motion vector in the solid, $\overline{\bar{\sigma}}$ is the stress tensor, and $\vec{f}$ is the source term. The ocean is considered an inviscid fluid with small displacement perturbations. The first-order momentum conservation and the continuity equation give the second-order scalar acoustic waves equation

$$
\rho_{f} \partial_{t}^{2} \vec{u}^{f}=-\vec{\nabla} p,
$$

where $\rho_{f}$ is the density of the fluid medium, $\vec{u}^{f}$ is the motion vector in the fluid, and $p$ is the acoustic pressure. Wave propagation in the ocean and the crust are coupled by the equality of the stresses applied on the fluid-solid interface

$$
\overline{\bar{\sigma}} \cdot \vec{n}=\partial_{t}^{2} \phi \cdot \vec{n},
$$

with $\phi$ such that $p=-\partial_{t}^{2} \phi$, and by the equality of the normal components of displacement at the interface

$$
\vec{u}^{s} \cdot \vec{n}=\vec{u}^{f} \cdot \vec{n} .
$$

SPECFEM3D integrates the weak form of these equations thanks to a high-order polynomial approximation (Tromp et al., 2008). The equations used in this code were first validated for seismology in a two-dimensional (2D) code (SPECFEM2D; Tromp et al., 2008), which was first applied and validated to marine acoustics by Cristini and Komatitsch (2012) and further tested for seismo-acoustic modeling (Bottero, 2018; Jamet et al., 2013). The 3D version of this code was first validated for seismology by Magnoni et al. (2014). We also verified that SPECFEM3D correctly handles horizontal refraction by performing a test as in Jensen et al. (2011) with a source in the water column above a truncatedwedge (as in Fig. 6.15 of Jensen et al., 2011). The results for a fluid-fluid (Pekeris waveguide) or solid-fluid model highlight the shadow zone due to horizontal refraction. ${ }^{1}$ SPECFEM2.5D also proved comparable to COMSOL in a similar wedge configuration (Bottero et al., 2016).

\section{B. Model parameters}

The aim is to simulate acoustic waves with a realistic behavior in simplified configurations highlighting 3D-effects. Preliminary simulations have shown that acoustic waves acquire their typical properties (e.g., sound-speed propagation) only beyond a distance of 50-100 km (Lecoulant et al., 2018). The calculation domain must also be large enough to set topographies with contrasting lateral extension, hence, causing contrasting 3D-effects. However, the size of the domain must be limited to keep the calculation time reasonable.

A domain $200 \times 50 \mathrm{~km}$ wide and $10 \mathrm{~km}$ thick provides a good compromise between these requirements (Fig. 1). It is vertically divided in a $3 \mathrm{~km}$ thick fluid medium (the ocean) underlain by a $7 \mathrm{~km}$ thick solid medium (the Earth's crust). All sides except the top sea-surface are perfectly matched absorbing layers (PML), which avoid unwanted reflections that would pollute the results (Xie et al., 2016). The crust, including the absorbing layer at its bottom, is thus considered as a semi-infinite medium. However, considering the speed of seismic waves and the frequency of the source given below, a typical $6 \mathrm{~km}$ thick ocean crust would be roughly a wavelength thick. Hence, the interface waves generated by seismic waves are probably affected by the choice of a semi-infinite crust, but not the acoustic waves on which this paper mainly focuses. The medium densities are set constant at typical values: $1000 \mathrm{~kg} \mathrm{~m}^{-3}$ in the water and $3200 \mathrm{~kg} \mathrm{~m}^{-3}$ in the Earth's crust. The model presented in this paper is a simple model that neglects the effects of refraction or sediments in spite of the large possible effect of the latter on the modal propagation of low-frequency acoustic waves (Ardhuin et al., 2013). The sound speed in the water $\left(1500 \mathrm{~m} \mathrm{~s}^{-1}\right)$ and the speed of $P$ waves $\left(5000 \mathrm{~m} \mathrm{~s}^{-1}\right)$ and $S$ waves $\left(3000 \mathrm{~m} \mathrm{~s}^{-1}\right)$ in the crust are set constant. These values are classical for $P$ and $S$ wave velocities in an oceanic crust (Searle, 2013). The refraction of sound waves in the Sound Fixing And Ranging (SOFAR) channel is known to be negligible for an ocean depth of $3 \mathrm{~km}$ in the band $2.8-21.2 \mathrm{~Hz}$ (Jamet et al., 2013). Furthermore, for the low-frequency signal used here $(\$ 2.5 \mathrm{~Hz})$, a $3 \mathrm{~km}$ thick ocean is only a few acoustic wavelengths thick $(\lessgtr 5)$, so the presence of a SOFAR channel would not matter. For the same purpose of focusing only on the effects of topography, there is no attenuation either in the solid or the fluid medium.

Two simplified topographies are used to test the generation of acoustic modes: a seamount and a linear ridge. An idealized seamount is built by rotating around the $z$ axis a Gaussian curve with a $2000 \mathrm{~m}$ height and $9 \mathrm{~km}$ half-width at half-height. A linear ridge is built by translating the same 

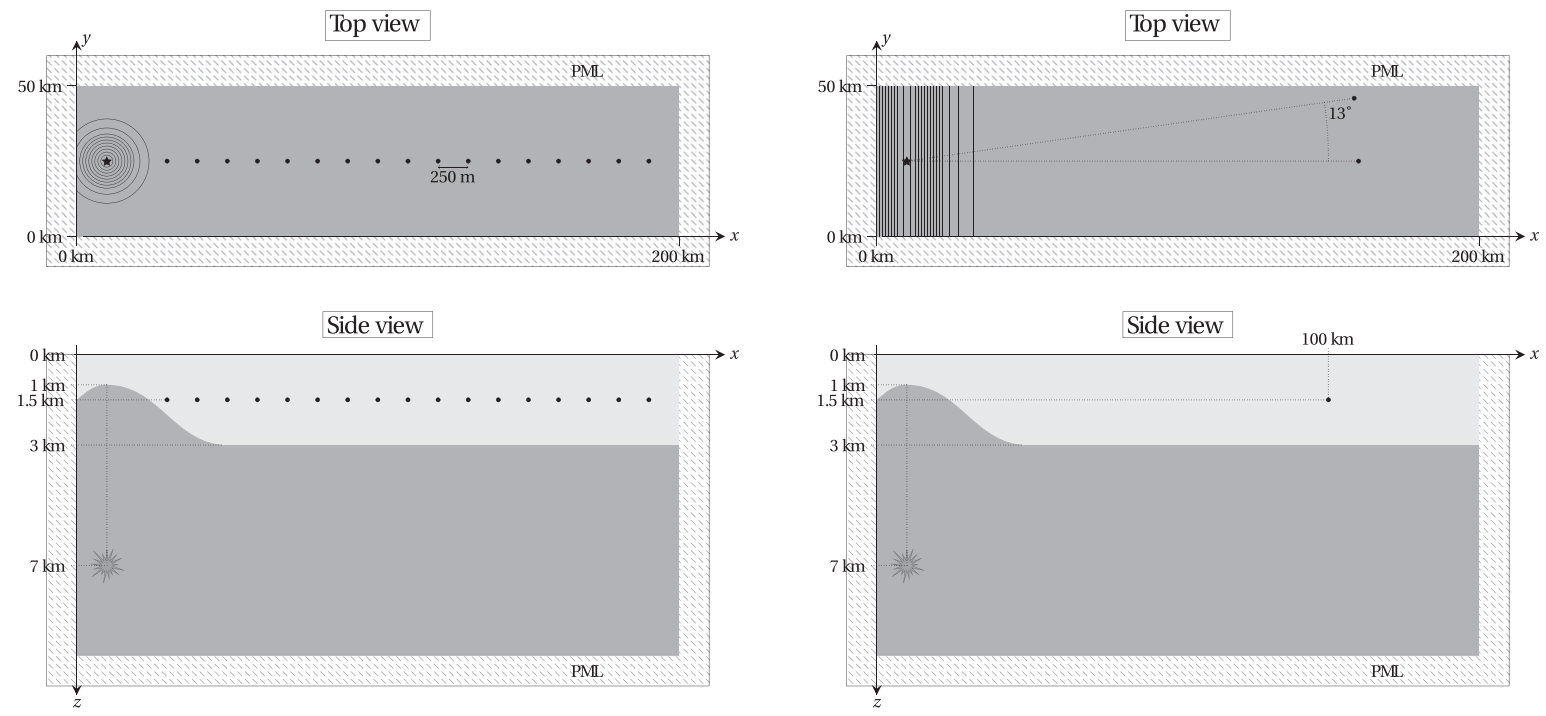

FIG. 1. Top and side views of the calculation domain (left) with a Gaussian seamount and (right) with a Gaussian linear ridge. Black lines are isobaths plotted every $100 \mathrm{~m}$; the flat bottom is $3000 \mathrm{~m}$ deep, and the topography rises up to $1000 \mathrm{~m}$ below the sea-surface. Black dots show the locations of the hydrophones where acoustic signals will be extracted; all hydrophones are at a depth of $1500 \mathrm{~m}$. The source (star) is at the apex of the seamount top or ridge crest. The hatched areas are the perfectly matched absorbing layers (PML; $2 \mathrm{~km}$ thick on the sides, $4 \mathrm{~km}$ thick at the bottom).

Gaussian cross section along the $y$ axis. These Gaussianshaped topographies have realistic sizes and are easy to model; they are comparable to the seamounts identified as the sources of $T$ waves signal in Chapman and Marrett (2006), with a rotational symmetry and a drop of $\sim 2000 \mathrm{~m}$ within $10 \mathrm{~km}$.

The source is located $4 \mathrm{~km}$ under the seafloor (i.e., $7 \mathrm{~km}$ below sea-surface), which is consistent with seismic events occurring in a seafloor spreading ridge context. It is simulated by a $0.5 \mathrm{~s}$ half-duration pseudo-Heaviside step function (Fig. 2). A pseudo-Heaviside signal is less usual than a Gaussian signal, but it is more realistic for near-field modeling. In any case, the elastic behavior of the solid medium turns the pseudo-Heaviside waveform into a Gaussian signal before it reaches the crust/ocean interface (Shearer, 2009). The focal mechanism is an isotropic explosion, which ensures that the acoustic pattern only reflects the effect of topography and not the radiation pattern of the source. The chosen seismic moment $\left(M_{0}=4.03 \times 10^{16} \mathrm{~N} \mathrm{~m}\right)$ corresponds to a medium magnitude earthquake $\left(M_{w}=5.0\right)$.
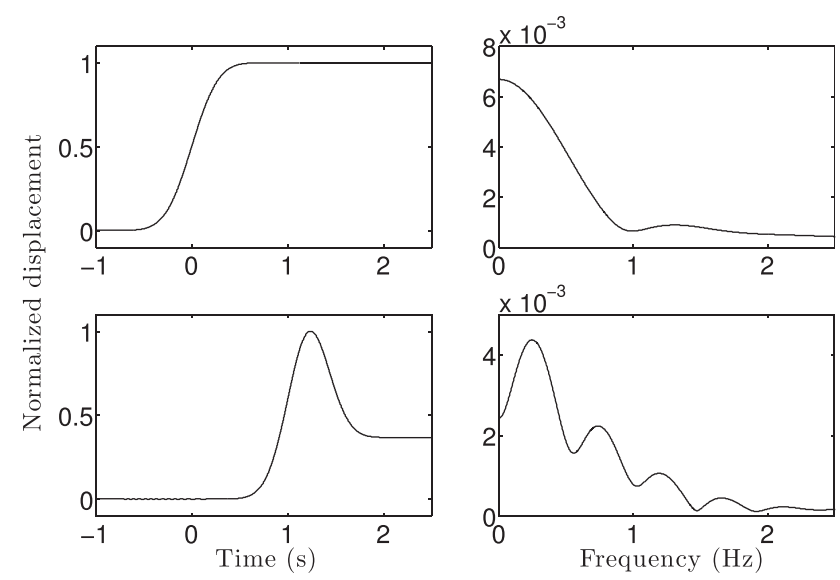

FIG. 2. Normalized displacement in the crust seen in the time-domain (left) and the frequency domain (right), at the source position (top) and $6 \mathrm{~km}$ above, at the top of the mount (bottom).
The appropriate way to mesh the calculation domain depends on the speeds of propagation of the waves in both media and on the frequency content of the signal. Indeed, to model waves with wavelength $\lambda$ with a spectral element method, a mesh with typical element size $\lambda$ is required, each element including five control points along each direction (Cristini and Komatitsch, 2012). Preliminary 2D simulations with the described source and an element size of $\sim 200 \mathrm{~m}$ show that the frequency content of the signal in the water column becomes negligible above $2.5 \mathrm{~Hz}$. Hence, a 3D-mesh with element size of $\sim 500 \mathrm{~m}$ in the fluid medium (lowest phase speed $\sim 1500 \mathrm{~m} \mathrm{~s}^{-1}$ ) is adequate. The domain is thus divided into 400 elements in the $x$-direction and 100 in the $y$ direction with their exact lateral extension being determined by the mesher. Vertically, there are six elements in the ocean and only ten in the Earth's crust with the larger velocities of seismic waves allowing larger spectral elements. Overall, the mesh comprises 560000 3D spectral elements and was built with the open-source software Gmsh (Geuzaine and Remacle, 2009). The five PML include four elements on the vertical edges and three elements on the bottom. After several tests, the dominant frequency for the calculation of the PML was chosen at $3 \mathrm{~Hz}$, ensuring the stability of the simulation and minimizing the noise level.

In such configuration, the typical run-time is on the order of $8 \mathrm{~h}$ on 336 parallel processors (12 cores), for a time signal of $200 \mathrm{~s}$ and a time-step of $1 \mathrm{~ms}$, which ensures the stability of the mesh for the considered element sizes and wave speeds.

\section{GENERATION AND PROPAGATION OF HYDROACOUSTIC WAVES}

\section{A. Analysis of the observed wavefield}

Waves produced in the water column by an earthquake can clearly be identified in a distance-time diagram [Fig. 3(top)]. This diagram is built from the time signals extracted on a linear array of hydrophones $1500 \mathrm{~m}$ deep and $250 \mathrm{~m}$ apart in the 

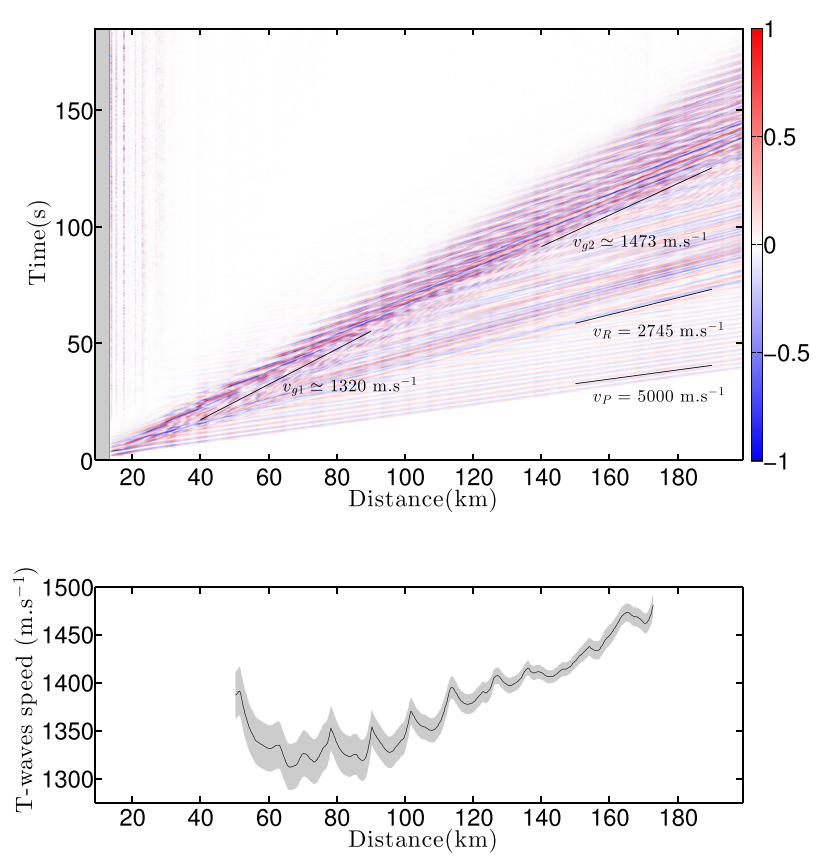

FIG. 3. (Color online) (Top) Distance-time diagram of the acoustic pressure normalized by its maximum at each hydrophone (i.e., distance) in the case of an isotropic explosion centered $4 \mathrm{~km}$ below a Gaussian seamount. $v_{P}$ is the speed of $P$-waves set in the model, $v_{R}$ is the Rayleigh waves speed without an ocean based on the $v_{P}$ and $v_{S}$ set in the model, and $v_{g 1}$ and $v_{g 2}$ are measured $T$-wave front speeds, respectively, between 40 and $90 \mathrm{~km}$ and between 140 and $190 \mathrm{~km}$. The grey area represents half the width of the seamount at the depth of the hydrophones $(1500 \mathrm{~m}$ below the sea-surface). (Bottom) Velocity of acoustic waves vs distance from the source, and the shaded area is the standard deviation.

symmetry plane of the domain (Fig. 1). In this distance-time diagram, the slope of the arrivals gives the speed of the waves. The first arrivals are $P$ waves propagating at the speed chosen for the model $\left(5000 \mathrm{~m} \mathrm{~s}^{-1}\right)$. They are followed by interface waves. The apparent speed of the first arrivals of these waves being $v_{R}=2745 \mathrm{~m} \mathrm{~s}^{-1}$, the theoretical phase speed of Rayleigh waves without an ocean, determined from $v_{P}$ and $v_{S}$ by

$$
v_{R}=v_{S} \frac{0.87+1.12 \nu}{1+\nu}
$$

(Viktorov, 1967), with $\nu$ being the Poisson ratio

$$
\nu=\frac{1}{2}\left[1-\frac{1}{\left(v_{P} / v_{S}\right)^{2}-1}\right] .
$$

The slowest and most energetic waves form a group propagating at a speed near the sound speed. To measure their velocity, faster waves were filtered out from the signals by removing all the energy propagating faster than $2500 \mathrm{~m} \mathrm{~s}^{-1}$ ( $P$ waves and interface waves) in a frequency-wavenumber domain [2D-Fourier transform, Fig. 4(top)]. Arrival times of the remaining waves were determined as the first time when the smoothed envelope of the filtered signal is half of its maximal amplitude. The speed of the first-arrival front is then obtained by a linear regression linking the position of the hydrophones to the arrival times. Figure 3(top) shows that those waves propagate first at a speed slower than the sound speed (e.g., $1289 \pm 24 \mathrm{~m} \mathrm{~s}^{-1}$ from 40 to $90 \mathrm{~km}$ ), which
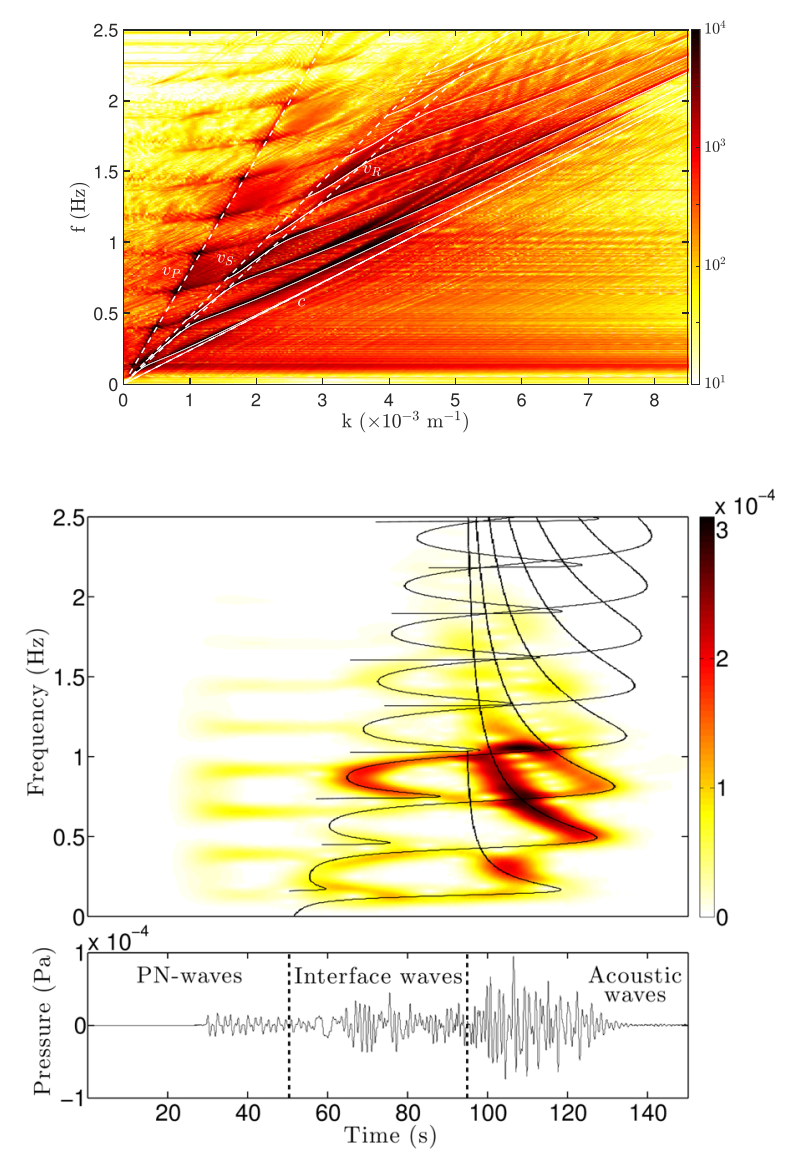

FIG. 4. (Color online) (Top) Frequency-wavenumber diagram of the normalized acoustic pressure showing the dispersion curves of simulated acoustic waves (colormap) and theoretical Rayleigh modes (white curves). Annotated white dashed lines show the phase speed of sound in water $(c)$, Rayleigh waves $\left(v_{R}\right), S$-waves $\left(v_{S}\right)$, and $P$ waves $\left(v_{P}\right)$. (Center) Spectrogram of a time signal measured by hydrophones $140 \mathrm{~km}$ away from the source at a depth of $1500 \mathrm{~m}$ (colormap) with the theoretical arrival times of Rayleigh modes depending on the frequency (black curves). (Bottom) Time series measured by the same hydrophone.

increases with the distance to approach the sound speed (e.g., $1480 \pm 7 \mathrm{~m} \mathrm{~s}^{-1}$ from 140 to $190 \mathrm{~km}$ ). This velocity is consistent with acoustic waves propagating in the water column. A linear regression of the acoustic modes arrivals with distance in $50 \mathrm{~km}$ long windows shows a regular increase of their velocity toward the sound speed [Fig. 3(bottom)]. The velocity scalloping is due to discontinuities in the acoustic front. As the acoustic waves propagate away from the source, the front becomes more continuous and their speed increases more regularly toward the sound speed.

The acoustic wavefield comprises several waves propagating at speeds higher than the speed of the first-arrival front. In a distance-time diagram [Fig. 3(top)], these waves appear with a slope lower than the wave front, which they finally cross before they vanish. Such behavior can be interpreted as a modal propagation. The higher speeds are the phase velocities of the acoustic waves. The lower speed of the first arrivals probably corresponds to the group velocity of the slowest mode. A widening acoustic wave-train, as it propagates, suggests an increasing modal dispersion.

Figure 4(top) compares the dispersion curves from the simulated results with the theoretical dispersion curves of 
Rayleigh modes in a finite depth ocean overlaying a semiinfinite elastic bottom (Abramovici, 1968; Ardhuin and Herbers, 2013). The energy is mostly located in the area corresponding to phase speeds between the sound speed and the speed of $S$ waves along the dispersion curves of Rayleigh modes. For a phase speed slightly smaller than $v_{R}$, Rayleigh modes reach an inflection point that separates the higher phase speed interface waves from the lower phase speed acoustic waves. Both interface waves and acoustic modes are pseudo-Rayleigh waves in different phase speed domains. $P N$ waves appear for phase speeds higher than the speed of $S$ waves with peaks along the line corresponding to the speed of $P$ waves.

The spectrogram [Fig. 4(center)] shows the successive arrivals of the three observed waves. First, $P N$ waves appear as lines parallel to the time-axis, reflecting the peaks located at discrete frequencies on Fig. 4(top). Second, interface waves appear along the curves showing the theoretical arrival times of Rayleigh modes with frequencies lower than the frequency of the turnaround point at the tail of the modal arrival. This turnaround point corresponds to a minimum of group velocity and to the inflection point of Rayleigh modes in the frequencywavenumber diagram [Fig. 4(top)]. Acoustic waves arrive only third, as their group velocity is necessarily lower than the sound speed. As interface waves, they are located along the theoretical curves of Rayleigh modes, but with frequencies higher than the one at the turnaround point.

\section{B. 3D-effects on the signal}

To highlight 3D-effects between a linear ridge and a seamount, we compared the time signals at two hydrophones located on a circle centered on the epicenter and $100 \mathrm{~km}$ away from the source at a depth of $1500 \mathrm{~m}$; one hydrophone is located in the symmetry plane of the model, and the other one on a line $13^{\circ}$ from this plane [Fig. 1(right)].

In the symmetry plane [Fig. 5(a)], the maximal amplitudes of the acoustic waves arrivals, $75 \mathrm{~s}$ after the beginning of the run, are about twice as large for the ridge case than the seamount case. The same amplitude difference is observed for the interface waves. The acoustic intensity received in the case of the ridge $\left(2.70 \times 10^{-3} \mathrm{~W} \mathrm{~m}^{-3} \mathrm{~s}^{-1}\right)$ is about five times larger than for the seamount $\left(5.84 \times 10^{-4} \mathrm{~W} \mathrm{~m}^{-2} \mathrm{~s}^{-1}\right)$. This can be explained by the larger lateral extension of the seismic/acoustic conversion zone for a linear ridge, the shallow zone being larger and hence the zone where acoustic modes are generated by seafloor scattering (de Groot-Hedlin and Orcutt, 1999). This larger zone can convert a larger amount of seismic energy into acoustic modes energy. There is also a small delay in the acoustic wave arrivals in the case of the ridge compared to the seamount. Half of the maximum of the signal envelope is reached $1.27 \mathrm{~s}$ later than in the seamount case, and the maximum of the envelope occurs $0.68 \mathrm{~s}$ later. This is probably due to the larger size of the conversion zone, leading to slightly longer acoustic paths.

At an angular distance $13^{\circ}$ away from the symmetry plane [Fig. 5(b)], the amplitudes are similar to the previous case as their difference in acoustic intensities $\left(2.60 \times 10^{-3}\right.$ $\mathrm{W} \mathrm{m} \mathrm{m}^{-2} \mathrm{~s}^{-1}$ in the ridge case vs $6.50 \times 10^{-4} \mathrm{~W} \mathrm{~m}^{-2} \mathrm{~s}^{-1}$ in
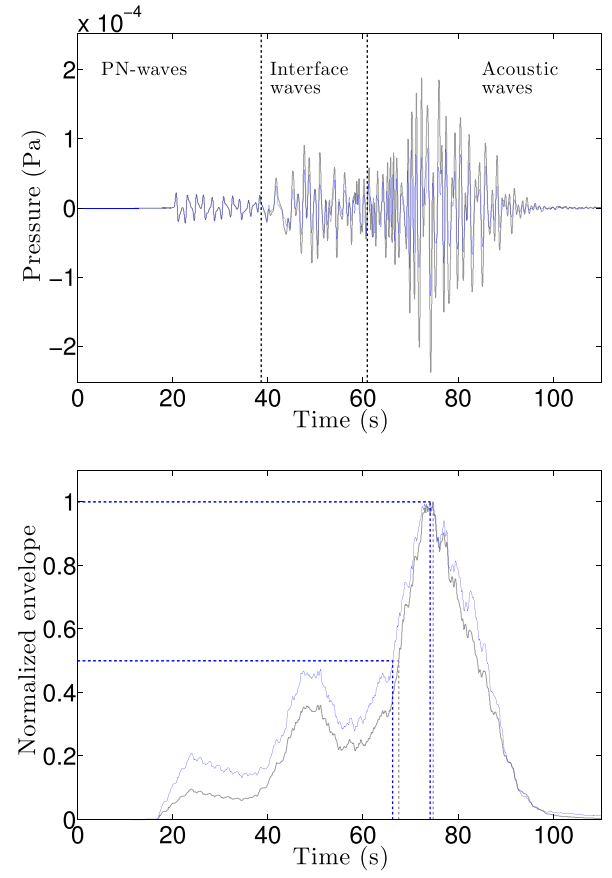

(a)
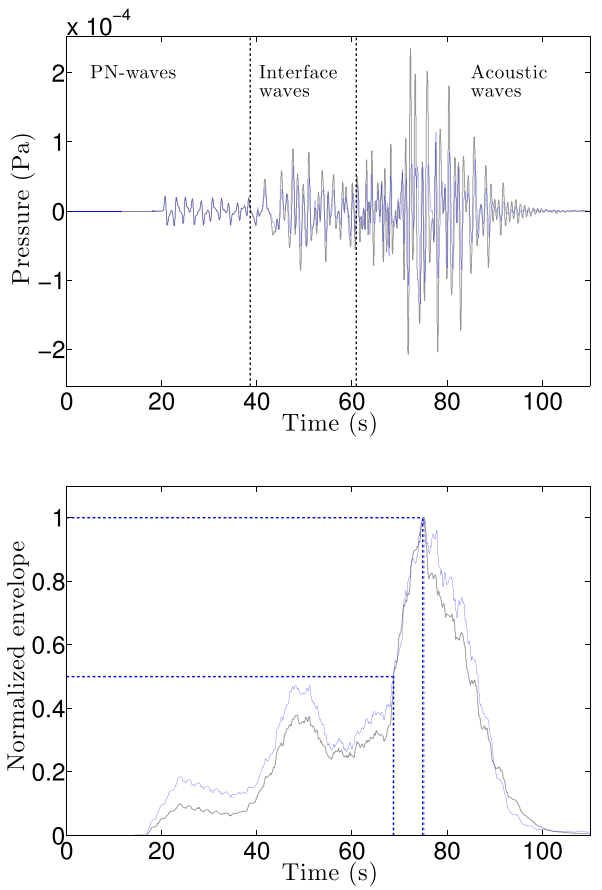

(b)

FIG. 5. (Color online) Time-signals and corresponding normalized envelopes for a Gaussian seamount (thin blue line) and a linear ridge (thick grey) measured by hydrophones $100 \mathrm{~km}$ away from the source (a) in the symmetry plane of the model and (b) on a line $13^{\circ}$ from the symmetry plane (see Fig. 1, right).

the seamount case). The delay in the arrival times is smaller: the maximum of the signal envelope occurs only $0.30 \mathrm{~s}$ later with the ridge than with the seamount, and the half of the maximum of the signal envelope is reached only $0.07 \mathrm{~s}$ later. Since the source is at the apex of the seamount, acoustic waves are radiating equally in all azimuths and any hydrophone on a circle centered on the epicenter will receive the 
same signal. However, in the case of the ridge, hydrophones at equal distance from the source but out of the symmetry plane will be slightly closer to the $T$ wave conversion area, which explains why the delay between $T$ wave arrivals almost disappears. Moreover, the signal results from the sum of acoustic waves generated first at near-source and later at far-source parts of the ridge. This explains the longer coda, still energetic, of the signal generated by the ridge, between 90 and $100 \mathrm{~s}$ after the beginning of the simulation [Fig. 5(b)]. In the symmetry plane, this effect on the coda between 90 and $100 \mathrm{~s}$ is hardly distinguishable [Fig. 5(a)].

\section{3D-EFFECTS ON THE LOCALIZATION OF AN EPICENTER}

\section{A. Source outside a hydrophone network}

In the context of monitoring spreading ridge seismicity with hydrophone networks, it is critically important to assess the accuracy of the localization of an earthquake epicenter with commonly used trilateration technics. Among the uncertainties, the bias introduced by the topography in the localization is not known. To address this question, we simulated a 28hydrophone network located at least $150 \mathrm{~km}$ from the source. The acoustic waves arrival times at each hydrophone are picked automatically, as in Sec. III A, except that, to avoid any interference from the $P$ waves and interface waves arrivals, the signal was zeroed prior to $95 \mathrm{~s}$. For a given selection of arrival times at selected hydrophones, we searched for the best location of the source with a probability density function (PDF) in a 2D-plane (Tarantola, 2005); the searched area is the whole $x$ $y$ domain (every $1 \mathrm{~km}$ ), and the propagation speed is assumed unknown and searched between $1000 \mathrm{~m} \mathrm{~s}^{-1}$ and $1600 \mathrm{~m} \mathrm{~s}^{-1}$. This interval is based on the measured $T$ wave speeds, which progressively increase up to the sound speed, but do not exceed it [Fig. 3(bottom)]. This approach resembles that used to locate $T$ wave acoustic sources from actual data (e.g., Fox et al., 2001), except that, here, the time origin of the event is known and equal to zero.

Figure 6 shows the results for a selected combination of four hydrophones; the best locations (highest probability density) do not exactly match the epicenter. In the seamount case, the best location is $4 \mathrm{~km}$ away from the epicenter in the $x$-direction on the upper slope of the seamount, and the best velocity is $1380 \mathrm{~m} \mathrm{~s}^{-1}$. In the ridge case, the best source location is $9 \mathrm{~km}$ southwest of the epicenter on the outer slope of the ridge, and the best velocity is $1430 \mathrm{~m} \mathrm{~s}^{-1}$. The crescent-shape uncertainty region reflects that the sourcereceiver distance is well estimated, whereas the source azimuth is poorly constrained due to the small geographical extension of the network and, hence, to the small differences in $T$ wave arrival times among the hydrophones (Tarantola, 2005). The 3D-effects highlighted in Sec. III B influence the source localization in two different ways. First, the crescentshape is located farther from receivers in the case of the ridge, due to an overall delay in the $T$ wave arrivals. Second, the highest probability density zone is shifted southwestward, due to lesser delays of acoustic waves at hydrophones far from the symmetry plane of the domain. If we use the same quadruplet, centered on the symmetry plane of the
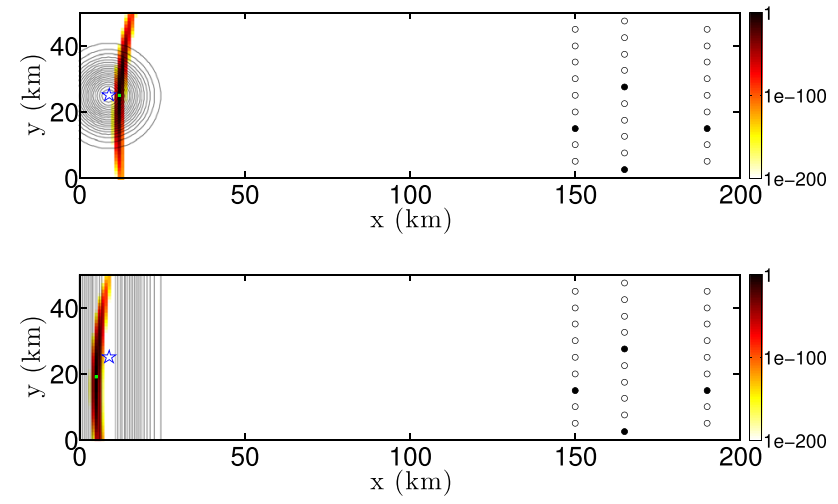

FIG. 6. (Color online) Normalized probability density of the acoustic source location based on the trilateration of arrival times at the four selected hydrophones (black dots) for (top) a Gaussian seamount and (bottom) a Gaussian ridge. The actual epicenter is represented by the blue star and the maximum of the probability density by the green square. Isobaths (black lines) are plotted every $100 \mathrm{~m}$, the flat bottom is at a depth of $3000 \mathrm{~m}$, and the topography rises up to $1000 \mathrm{~m}$ below the sea-surface. Open dots show the location of all the hydrophones inverted to produce Fig. 7.

domain (keeping the same $x$ coordinates), the second effect vanishes, and the probability density for the seamount and the ridge only differ by the distance from the receivers.

To fully evaluate the bias caused by the topography in the localization of the acoustic source, we repeated the previous experiment for each of the 20475 quadruplets of hydrophones among the 28 hydrophones of our network (open circles in Fig. 6). For a given quadruplet, the location of the source is identified by the maximum of the PDF. All maxima falling on the domain boundaries are ignored, as well as all solutions in which the best velocity lies outside the imposed speed range. PDF maxima are then sorted in a histogram with 20475 bins, and all solutions in the bin with the lowest PDF value are ignored (i.e., considered as the least reliable solutions of all trilaterations). Figure 7 displays the number of times a grid node has been found as the most probable source location. Reassuringly, the resulting patterns are symmetrical relative to the symmetry axis of the model. In the seamount case, the majority of source locations are near the top of the seamount within a distance of $5 \mathrm{~km}$ from the epicenter [Fig. 7(top)]. The standard deviation of the distance from the source projected on the $y$ axis, given the azimuthal dispersion of the solutions, is $4 \mathrm{~km}$. In the linear ridge case, the majority of locations cluster in two areas parallel to the ridge orientation: near the ridge crest and within $1 \mathrm{~km}$ of the epicenter, and along the upper outer slope of the ridge, $3-4 \mathrm{~km}$ away from the epicenter [Fig. 7(bottom)]. The denser number of solutions on the outer slope of the ridge probably derives from the delay of acoustic waves generated with the ridge compared to acoustic waves emitted by the seamount. This delay leads to an overestimation of the distance between the source and the receivers. The standard deviation for the latter area projected on the $y$ axis is $6 \mathrm{~km}$, reflecting its larger lateral extension. In both cases, the distribution of the most probable solutions provides an approximation of the shape and extent of the seismo-acoustic conversion zone.

In both cases, the best-fitting sound speeds in the trilateration range from 1300 to $1500 \mathrm{~m} \mathrm{~s}^{-1}$ with a maximum at 

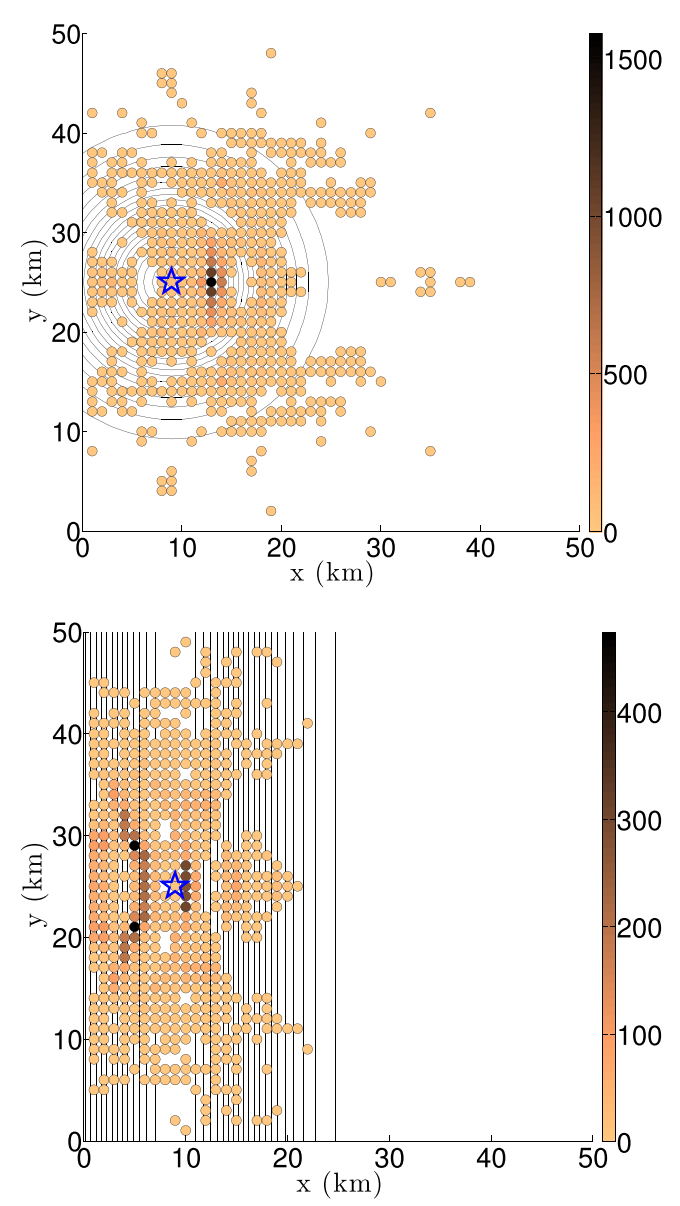

FIG. 7. (Color online) Most probable locations for the acoustic source by trilateration for (top) a Gaussian seamount and (bottom) a Gaussian linear ridge. The color scale corresponds to the number of times a grid node has been found as the most probable location for all quadruplets of hydrophones among the 28 shown in Fig. 6 . The calculation domain is here zoomed in a $50 \times 50 \mathrm{~km}$ area with the topography and the epicenter (blue star). Isobaths (black lines) are plotted every $100 \mathrm{~m}$, the flat bottom is at a depth of $3000 \mathrm{~m}$, and the topography rises to $1000 \mathrm{~m}$ below the sea-surface.

$1370 \mathrm{~m} \mathrm{~s}^{-1}$ in the case of the mount [Fig. 8(top)] and at $1430 \mathrm{~m} \mathrm{~s}^{-1}$ in the case of the ridge [Fig. 8(bottom)]. The difference with the theoretical speed $\left(1500 \mathrm{~m} \mathrm{~s}^{-1}\right)$ is the result of a lower speed of acoustic waves at the beginning of their propagation (Fig. 3). The ridge case shows more dispersion in the optimal sound speeds from the trilaterations, which can be linked to a larger conversion zone. Indeed, all arrival times are calculated from the beginning of the simulation, assuming that all acoustic waves are emitted at once. This approximation is sensible for acoustic waves emitted in the vicinity of the source. However, acoustic waves generated far from the source are emitted later since seismic waves need to propagate over a longer distance until the conversion zone. Assuming they have been emitted at the same time as the near-source acoustic waves underestimates their speed.

\section{B. Source inside a hydrophone network}

The location uncertainties pointed out in Sec. IV A partly came from the fact that the source was outside the hydrophone network, which is known as the least favorable configuration. Actual hydrophone networks are normally
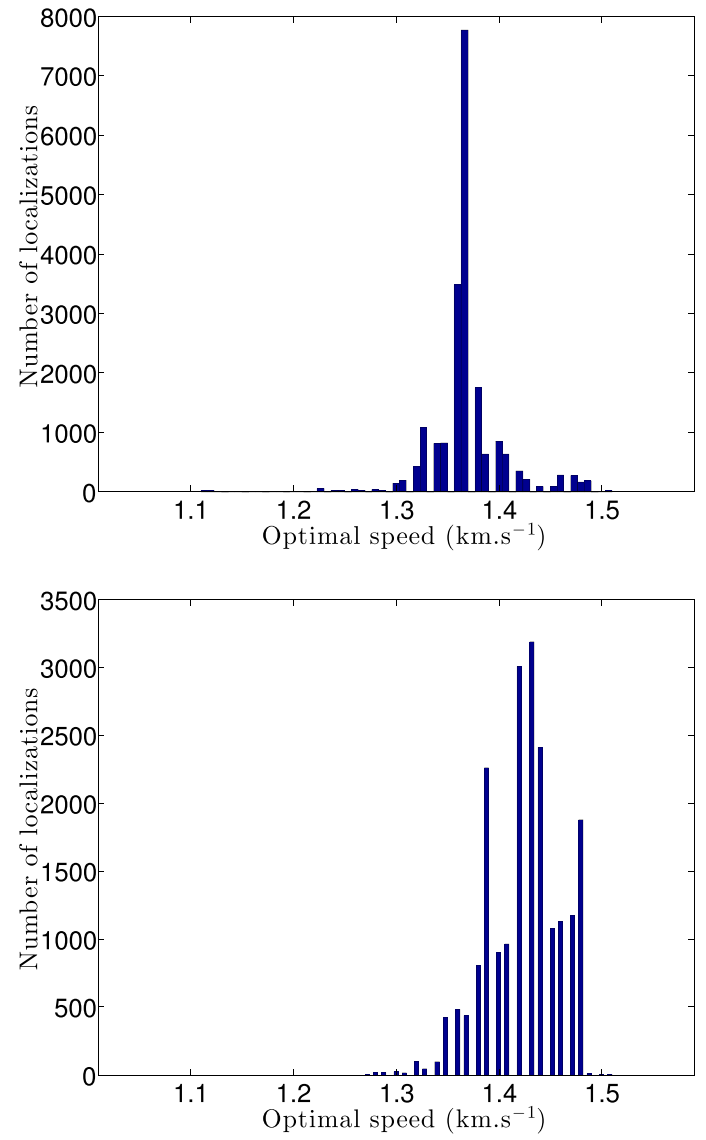

FIG. 8. (Color online) Histogram of the acoustic sound speed matching the maximum probability density for a given acoustic source location in the case of (top) a Gaussian seamount and (bottom) a Gaussian linear ridge.

deployed on both sides of seafloor spreading ridges to ensure that earthquakes occur within the hydrophone network. To test such configuration, we created hydrophones on the other side of the source with the same $y$ coordinates as before and $x$ coordinates symmetrical relative to the $O y$-plane containing the source; hence, we can use the same arrival times. Figure 9 shows the results for a selected combination of four hydrophones on either side of the source with three hydrophones identical to those in the experiment in Sec. IV A and the fourth one replaced by its symmetric across the relief.

The best locations (highest probability density) do not exactly match the epicenter. In the seamount case, the best location is $4 \mathrm{~km}$ away from the epicenter in the $y$-direction on the upper slope of the seamount, and the best velocity is $1410 \mathrm{~m} \mathrm{~s}^{-1}$. In the ridge case, the best source location is $7 \mathrm{~km}$ south of the epicenter on the crest of the ridge, and the best velocity is $1390 \mathrm{~m}$ $\mathrm{s}^{-1}$. The uncertainty region is a narrow strip along the $O y$-plane containing the source. Hydrophones on both sides better constrain the distance of the source from the receivers, narrowing the uncertainty region. The azimuth is also better constrained, but still less than the distance between the source and the receivers. As previously, 3D-effects appear as a difference in the azimuth between the seamount and the ridge. In the case of the ridge, the best source location is shifted southward, under the influence of acoustic waves emitted far from the source.

To fully evaluate the bias caused by the topography for a source inside a hydrophone network, we repeated the 

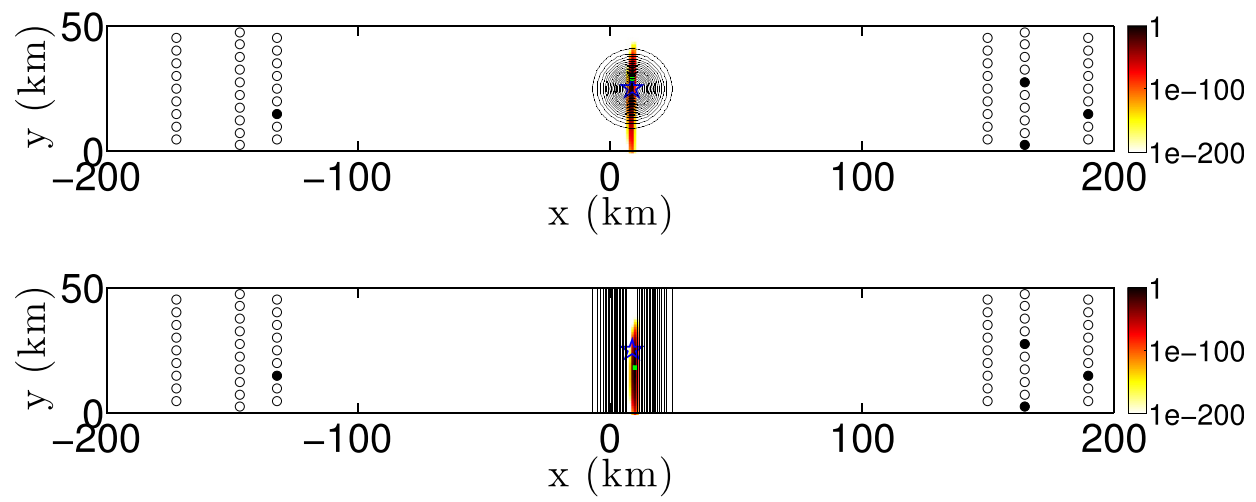

FIG. 9. (Color online) Normalized probability density of the acoustic source location based on the trilateration of the arrival times at the four selected hydrophones (black dots) for (top) a Gaussian seamount and (bottom) a Gaussian ridge. The actual epicenter is represented by the blue star and the maximum of the probability density by the white square. Isobaths (black lines) are plotted every $100 \mathrm{~m}$, the flat bottom is at a depth of $3000 \mathrm{~m}$, and the topography rises up to $1000 \mathrm{~m}$ below the sea-surface. Open dots show the location of all the hydrophones inverted to produce Fig. 10.

previous experiment for each of the 367290 quadruplets of hydrophones among the 56 hydrophones of the built network (void circles in Fig. 9). Figure 10(a) displays the number of times a grid node has been found as the most probable source location, only taking into account quadruplets with at least one hydrophone on each side. In both cases, the source is located in an area centered on the epicenter, very narrow in the $x$ direction (less than $3 \mathrm{~km}$ ) and elongated in the $y$-direction ( $7 \mathrm{~km}$ in the seamount case vs $14 \mathrm{~km}$ long in the ridge case).

Despite the differences in the localization methods, these values can be compared to experimental uncertainties on the location of acoustic events. The one standard deviation

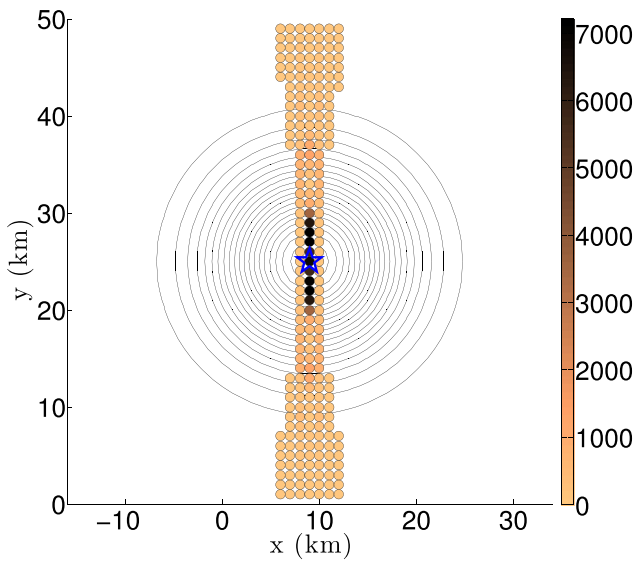

(a)

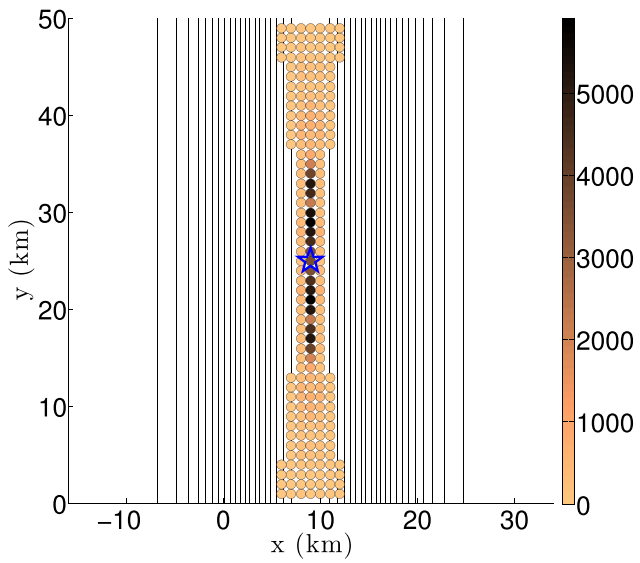

(c)

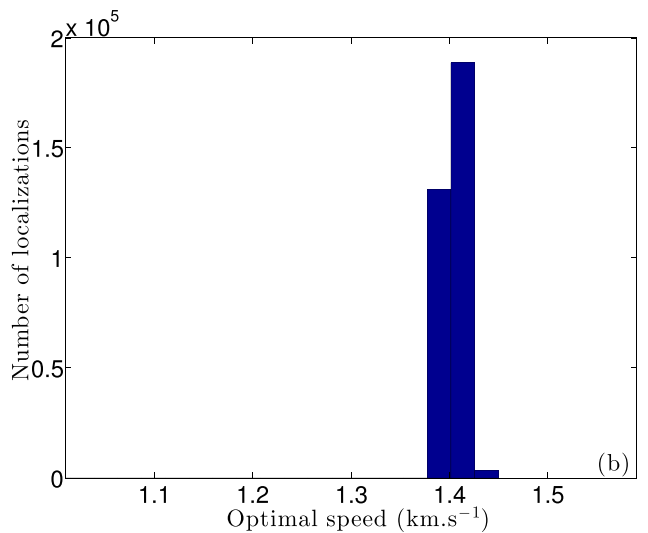

(b)

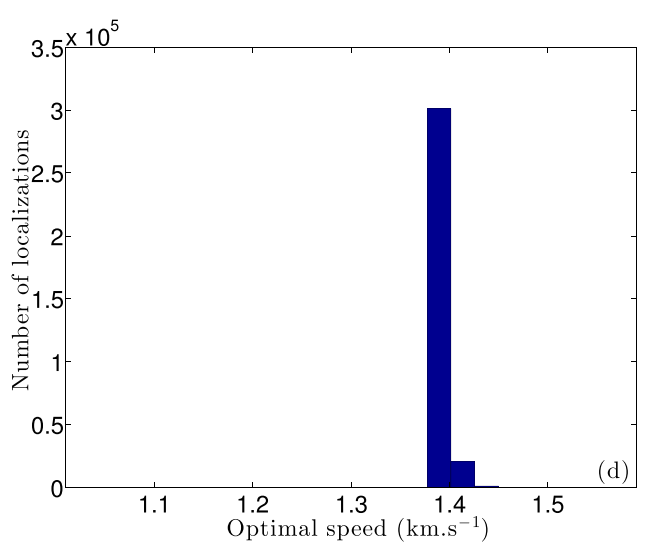

(d)

FIG. 10. (Color online) (a),(c) Most probable locations for the acoustic source by trilateration for (a) a Gaussian seamount and (c) a Gaussian linear ridge with hydrophones on either side of the source. (b),(d) Corresponding histograms of the best sound speed matching the maximum probability density in the case of (b) a Gaussian seamount and (d) a Gaussian linear ridge. 
uncertainty in latitude or longitude are, for instance, less than $5 \mathrm{~km}$ inside an array of hydrophones of $\sim 1000 \times \sim 1000 \mathrm{~km}$ (e.g., Fox et al., 2001; Royer et al., 2015; Smith et al., 2003; Tsang-Hin-Sun et al., 2016) up to $10 \mathrm{~km}$ inside a smaller array of $\sim 500 \times \sim 500 \mathrm{~km}$ (Giusti et al., 2018). They can be smaller by a factor of 2 depending on the number of hydrophones used in the inversion (from four to six). However, these trilaterations also search for the time origin of the event, with one standard deviation uncertainty in the order of 2-5s. Our results suggest that either the experimental uncertainties are underestimated since they overlook any effect of the bathymetry or, conversely, that the errors due to the topography are less influential at ranges larger than $200 \mathrm{~km}$ (i.e., the errors on the arrival times at the hydrophones then include the topography effects). The robustness of the location determination from large arrays of hydrophones favors the latter hypothesis. At smaller ranges, the errors due to the topography may prevail upon the propagation errors and should be taken into account. But this preponderance has yet to be tested in realistic cases.

Using hydrophones on both sides of the source better constrains the distance of the source from the lines of hydrophones than its azimuth. This 3D-effect is likely due to the larger seismic/acoustic conversion zone in the ridge case, although we cannot preclude a combined effect with the peculiar distribution of the hydrophones parallel to the ridge crest. In both cases, the best sound speeds found by trilateration [Figs. 10(b) and 10(d)] are much less dispersed than when the source is outside the hydrophone network (Fig. 8). Moreover, they are both centered near $1400 \mathrm{~m} \mathrm{~s}^{-1}$ : $1390 \mathrm{~m}$ $\mathrm{s}^{-1}$ in the seamount case vs $1410 \mathrm{~m} \mathrm{~s}^{-1}$ in the ridge case [Fig. 8(a)]. These values are still lower than $1500 \mathrm{~m} \mathrm{~s}^{-1}$, probably for the same reasons as stated in Sec. IV A. The slow start of acoustic waves at the beginning of their propagation may bias the location determination when the source is close to a hydrophone network (distance less than $100 \mathrm{~km}$ ), if one assumes a constant sound speed.

\section{Source away from a seamount}

Hydroacoustic recordings show that the regions where $T$ waves are excited by downslope propagation do not necessarily coincide with the earthquake epicenter (Chapman and Marrett, 2006; Johnson and Norris, 1968). To test this observation in a simplified configuration, the source of the earthquake is translated along $y, 20 \mathrm{~km}$ away from the summit of the Gaussian seamount under a flat-bottom area. The localizations from the 20475 quadruplets of hydrophones in the 28hydrophone network are shown Fig. 11(top). The number of localizations is significant in three main areas: first, in the flat area near the epicenter, second along the foot-slope of the seamount near the epicenter, and third at the foot of the seamount far from the epicenter. The latter two areas are consistent with acoustic waves generated by downslope propagation away from the source. The former area is consistent with the generation of acoustic waves in spite of a flat bottom, those waves being known for their conversion zone coincident with the epicenter (Johnson et al., 1968). However, since all solutions falling on the model boundaries, or outside the sound-speed range, or with a too small probability density have been
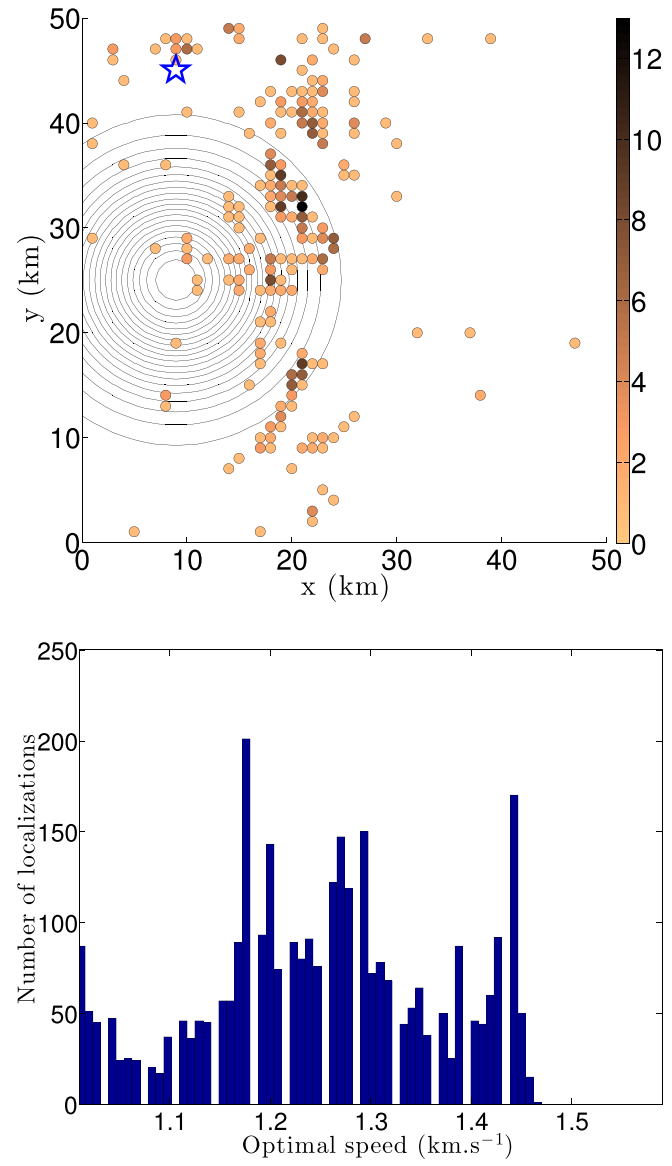

FIG. 11. (Color online) (Top) Location determinations of the epicenter by trilateration (colored points by number of localizations) in the case of a Gaussian seamount with a source $20 \mathrm{~km}$ away from its summit (blue star). Zoom on the $50 \times 50 \mathrm{~km}$ relief zone, isobaths (black lines) are plotted every $100 \mathrm{~m}$, the flat bottom is at a depth of $3000 \mathrm{~m}$, and the topography rises up to $1000 \mathrm{~m}$ below the sea-surface. (Bottom) Corresponding histogram of the best sound speeds matching the maximum probability density.

discarded, the most probable locations (i.e., number of localizations per point) are only based on 424 solutions. The soundspeed distribution [Fig. 11(bottom)] is far more dispersed than in the previous cases (Fig. 8). Again, this observation can be explained by acoustic waves being emitted at delayed times, depending on the location of the seismo-acoustic conversion relative to the hydrophone location.

Finally, with hydrophones on either side of the source (Fig. 12), the most probable locations are found near the epicenter ( $4 \mathrm{~km}$ away) on the flat part (i.e., abyssal plain) of the model. Other solutions spread in the vicinity of the epicenter or on the slopes of the mount. The source determination is definitely improved when the source is inside a hydrophone network, but the emission of acoustic waves by nearby topographic slopes may introduce a bias in the solution. The best sound speeds [Fig. 12(bottom)] show much less dispersion than when the source is outside the hydrophone network, reflecting the better constraints on the distance between the source and the receivers.

\section{DISCUSSION}

This paper first demonstrates that SPECFEM3D is a very convenient tool to model the generation of very lowfrequency acoustic waves on hilly sea-bottom and abyssal 

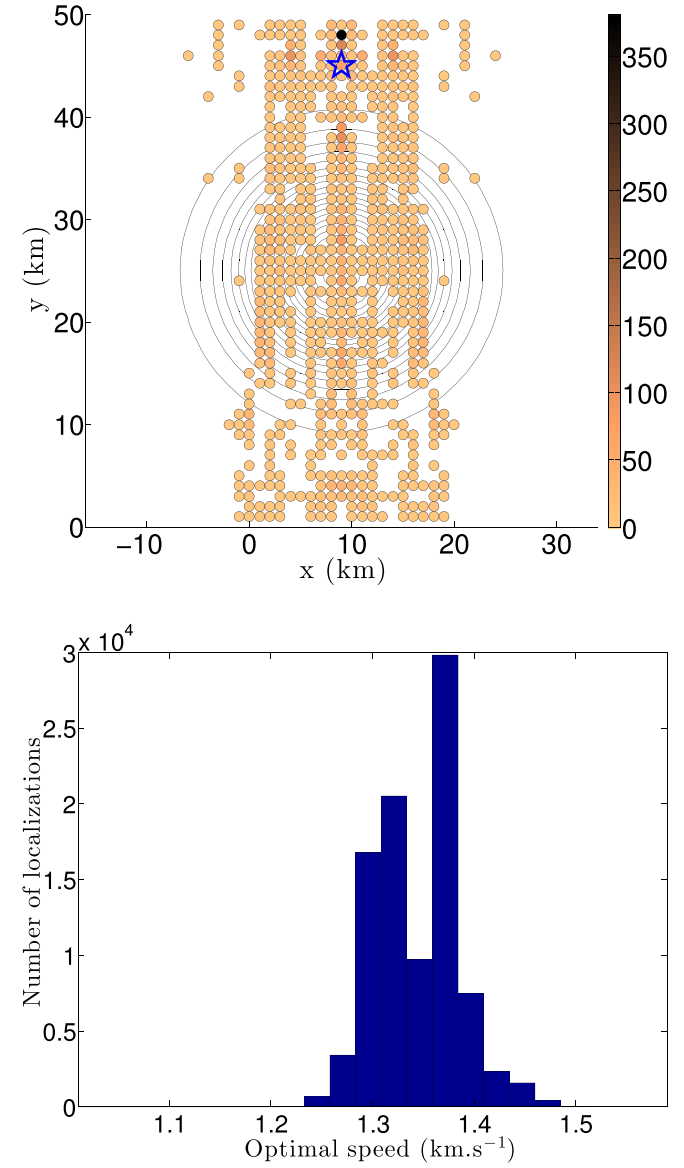

FIG. 12. (Color online) (Top) Most probable locations for the acoustic source by trilateration with a source away from a Gaussian seamount and with hydrophones on either side. (Bottom) Corresponding histogram of the best sound speeds matching the maximum probability density.

plains. These acoustic waves are shown to propagate as Rayleigh modes and are affected by modal dispersion.

Second, our simulations highlight different 3D-effects in the generation of those very low-frequency acoustic waves by a source located beneath a Gaussian seamount or a Gaussian ridge. Significant differences are observed in the amplitude of simulated signals, more powerful in the ridge case, which means that the acoustic source-level of an earthquake may not only reflect its magnitude but also the size, orientation, and slope of the conversion area. There are also clear 3D topographic effects in the acoustic wave arrival times at hydrophones, which may bias the location determination of an event by trilateration by several kilometers. For example, due to its larger seismic/acoustic conversion zone, a linear ridge may introduce larger uncertainties in the source location (up to $14 \mathrm{~km}$ ). However, our simulations are limited to a domain $200 \mathrm{~km}$ long (i.e., arrival times in the order of $60-80 \mathrm{~s}$ ); it is likely that the observed delays (smaller than $1 \mathrm{~s}$ ) will become negligible when hydrophones are several hundred kilometers away from a source. More importantly, we show that, in the presence of topography, arrival-time inversions may point to the slopes of a topographic feature, which may be a more efficient $T$ wave radiator than the epicenter itself.

The 3D-effects in our simulations are strongly analogous to the ones observed for $T$ waves (e.g., Chapman and Marrett, 2006; de Groot-Hedlin and Orcutt, 1999). However,

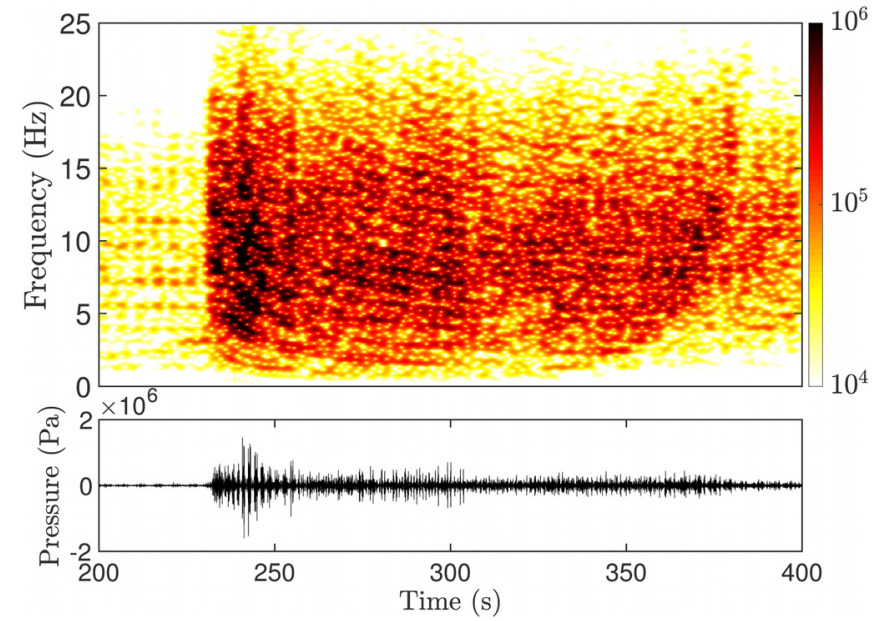

FIG. 13. (Color online) Spectrogram of a time-signal simulated by SPECFEM2D at a depth of $1000 \mathrm{~m}, 350 \mathrm{~km}$ away from a $10 \mathrm{~Hz}$ source, and propagating in an homogeneous ocean layer overlaying an homogeneous crust (Jamet, 2014).

due to the difference of frequency $(0-2.5 \mathrm{~Hz}$ instead of $4-40 \mathrm{~Hz}$ for $T$ waves), further work is needed on the modal propagation of the acoustic waves simulated by SPECFEM3D, particularly in higher frequency. 2D simulations (SPECFEM2D; Jamet, 2014) with a $10 \mathrm{~Hz}$ source generate a signal up to $25 \mathrm{~Hz}$ with modes similar to our 3D simulations with a $1 \mathrm{~Hz}$ source (Fig. 13 vs Fig. 4, center). Note the 2.5 factor between the frequency of the source and the maximal frequency observed in the water column. Additional tests at higher frequencies show the occurrence of Rayleigh modes at frequencies up to $25 \mathrm{~Hz}$, even with a SOFAR channel. ${ }^{1}$ This suggests that SPECFEM3D would behave at $T$ wave frequencies in a similar way to here with very low frequencies. Further work needs also to include more complex topography (e.g., from a swath-bathymetry survey) and simulations of $T$ wave at longer distances $(500 \mathrm{~km})$, which will require calculation facilities able to handle very large meshes $(50 \times 500 \times 20 \mathrm{~km})$. As an alternative, one can consider highresolution 3D models near the source, and use the outputs at its edges as inputs in a 2D-model for propagating $T$ waves over longer distances.

\section{ACKNOWLEDGMENTS}

J.L. was supported by a joint Ph.D. grant from the Délégation Générale de l'Armement (DGA) and the Région Bretagne. The SPECFEM3D code was run on Datarmor, a massive computing facility common to several research institutions at the westernmost tip of Brittany (France). The authors are grateful to Cédric Ody at Institut Universitaire Européen de la Mer (IUEM) for solving meshing errors, Charles Peureux for the understanding of the modal propagation, and to two anonymous referees whose helpful comments have greatly improved the paper.

\footnotetext{
${ }^{1}$ See supplementary material at https://doi.org/10.1121/1.5126009 for a classical benchmark test of horizontal refraction with SPECFEM3D and showing the occurrence of energetic Rayleigh modes at frequencies representative of actual $\mathrm{T}$ waves $(25 \mathrm{~Hz})$, even in a model with a SOFAR channel.
}

Abramovici, F. (1968). "Diagnostic diagrams and transfer functions for ocean waveguides," Bull. Seismol. Soc. Am. 58(1), 427-456. 
Ardhuin, F., and Herbers, T. H. C. (2013). "Noise generation in the solid Earth, oceans and atmosphere, from nonlinear interacting surface gravity waves in finite depth," J. Fluid Mech. 716, 316-348.

Ardhuin, F., Lavanant, T., Obrebski, M., Mari, L., Royer, J.-Y., d'Eu, J.-F., Howe, B. M., Lukas, R., and Aucan, J. (2013). "A numerical model for ocean ultra-low frequency noise: Wave-generated acoustic-gravity and Rayleigh modes," J. Acoust. Soc. Am. 134(4), 3242-3259.

Bohnenstiehl, D., Tolstoy, M., Dziak, R., Fox, C., and Smith, D. (2002). "Aftershock sequences in the mid-ocean ridge environment: An analysis using hydroacoustic data," Tectonophysics 354(1-2):49-70.

Bottero, A. (2018). "Full-wave numerical simulation of $T$-waves and of moving acoustic sources," Ph.D. thesis, Université Aix Marseille, available at https://tel.archives-ouvertes.fr/tel-01893011 (Last viewed 9/9/2019).

Bottero, A., Cristini, P., Komatitsch, D., and Asch M. (2016). “An axisymmetric time-domain spectral-element method for full-wave simulations: Application to ocean acoustics," J. Acoust. Soc. Am. 140(5), 3520-3530.

Chapman, N. R., and Marrett, R. (2006). "The directionality of acoustic Tphase signals from small magnitude submarine earthquakes," J. Acoust. Soc. Am. 119(6), 3669-3675.

Cristini, P., and Komatitsch, D. (2012). "Some illustrative examples of the use of a spectral-element method in ocean acoustics," J. Acoust. Soc. Am. 131(3), 229-235.

de Groot-Hedlin, C. D., and Orcutt, J. A. (1999). "Synthesis of earthquakegenerated T-waves," Geophys. Res. Lett. 26(9), 1227-1230, https://doi.org/ $10.1029 / 1999 \mathrm{~g} 1900205$.

Fox, C. G., Matsumoto, H., and Lau, T.-K. A. (2001). "Monitoring Pacific Ocean seismicity from an autonomous hydrophone array," J. Geophys. Res.: Solid Earth 106(B3), 4183-4206, https://doi.org/10.1029/2000JB900404.

Geuzaine, C., and Remacle, J.-F. (2009). "Gmsh: A 3-D finite element mesh generator with built-in pre- and post-processing facilities," Int. J. Numer. Meth. Eng. 79(11), 1309-1331.

Giusti, M., Perrot, J., Dziak, R. P., Sukhovich, A., and Maia, M. (2018). "The August 2010 earthquake swarm at North FAMOUSFAMOUS segments, Mid-Atlantic Ridge: Geophysical evidence of dike intrusion," Geophys. J. Int. 215(1), 181-195.

Jamet, G. (2014). "Modélisation d'ondes sismo-acoustiques par la méthode des éléments spectraux. Application à un séisme dans l'Atlantique Nord" ("Modeling of sismo-acoustic waves by a spectral element method. Application to an earthquake in the North Atlantic"), Ph.D. thesis, Université de Bretagne Occidentale, Brest, available in French at https:// tel.archives-ouvertes.fr/tel-01091245 (Last viewed 9/9/2019).

Jamet, G., Guennou, C., Guillon, L., Mazoyer, C., and Royer, J.-Y. (2013).

" $T$-wave generation and propagation: A comparison between data and spectral element modeling,” J. Acoust. Soc. Am. 134(4), 3376-3385.

Jensen, F. B., Kuperman, W. A., Porter, M. B., and Schmidt, H. (2011). Computational Ocean Acoustics, 2nd ed., in Modern Acoustics and Signal Processing (Springer, New York).

Johnson, R. H., and Norris, R. A. (1968). " $T$-phase radiators in the western Aleutians," Bull. Seismol. Soc. Am. 58(1), 1-10.

Johnson, R. H., Norris, R. A., and Duennebier, F. K. (1968). "Abyssally Generated T Phases," in The Crust and Upper Mantle of the Pacific Area,
Geophys. Monogr. Series, edited by L. Knopoff, C. L. Drake, and P. J. Hart (American Geophysical Union, Washington, D.C.) Vol. 12, pp. 70-78.

Lecoulant, J., Guennou, C., Guillon, L., and Royer, J.-Y. (2018). "Modélisation 3D des ondes acoustiques (ondes $T$ ) générées par les séismes sous-marins" ["3D modeling of acoustic waves (T waves) generated by submarine earthquakes"], in Actes du 14e Congrès Français d'Acoustique (Proceedings of the 14th French Congress of Acoustics) (Le Havre, France), pp. 339-343, available in French at https://www.conforg.fr/ cfa2018/output_directory2/data/articles/000136.pdf (Last viewed 9/19/ 2019).

Magnoni, F., Casarotti, E., Michelini, A., Piersanti, A., Komatitsch, D., Peter, D., and Tromp, J. (2014). "Spectral-element simulations of seismic waves generated by the 2009 L'Aquila earthquake," Bull. Seismol. Soc. Am. 104(1), 73-94.

Okal, E. A. (2008). "The generation of $T$ waves by earthquakes," in Advanced Geophysics (Elsevier, Amsterdam), Vol. 49, pp. 1-65.

Peter, D., Komatitsch, D., Luo, Y., Martin, R., Le Goff, N., Casarotti, E., Le Loher, P., Magnoni, F., Liu, Q., Blitz, C., Nissen-Meyer, T., Basini, P., and Tromp, J. (2011). "Forward and adjoint simulations of seismic wave propagation on fully unstructured hexahedral meshes: SPECFEM3d Version Sesame," Geophys. J. Int. 186(2), 721-739.

Royer, J.-Y., Chateau, R., Dziak, R., and Bohnenstiehl, D. (2015). "Seafloor seismicity, Antarctic ice-sounds, cetacean vocalizations and long-term ambient sound in the Indian Ocean basin," Geophys. J. Int. 202(2), $748-762$.

Searle, R. (2013). Mid-Ocean Ridges (Cambridge University Press, Cambridge, UK).

Shearer, P. M. (2009). Introduction to Seismology, 2nd ed. (Cambridge University Press, Cambridge, UK).

Smith, D. K., Escartin, J., Cannat, M., Tolstoy, M., Fox, C. G., Bohnenstiehl, D. R., and Bazin, S. (2003). "Spatial and temporal distribution of seismicity along the northern Mid-Atlantic Ridge $\left(15^{\circ}-35^{\circ} \mathrm{N}\right)$," J. Geophys. Res.: Solid Earth 108(B3), 2167-2188, https://doi.org/ 10.1029/2002JB001964

Tarantola, A. (2005). Inverse Problem Theory and Methods for Model Parameter Estimation (Society for Industrial and Applied Mathematics, Philadelphia, PA).

Tromp, J., Komatitsch, D., and Qinya, L. (2008). "Spectral-element and adjoint methods in seismology," Commun. Comput. Phys. 3(1), 1-32.

Tsang-Hin-Sun, E., Royer, J.-Y., and Perrot, J. (2016). "Seismicity and active accretion processes at the ultraslow-spreading Southwest and intermediate-spreading Southeast Indian ridges from hydroacoustic data," Geophys. J. Int. 206(2), 1232-1245.

Viktorov, I. A. (1967). Rayleigh and Lamb Waves Physical Theory and Applications (Springer, New York).

Xie, Z., Matzen, R., Cristini, P., D. Komatitsch, and Martin, R. (2016). “A perfectly matched layer for fluid-solid problems: Application to oceanacoustics simulations with solid ocean bottoms," J. Acoust. Soc. Am. 140(1), 165-175. 